\title{
Phase and Amplitude Control Ability using Spatial Light Modulators and Zero Path Length Difference Michelson Interferometer
}

\author{
Michael G. Littman, Michael Carr ${ }^{\perp}$, Jim Leighton, Ezekiel Burke, David Spergel ${ }^{\perp}$ and Jeremy
}

Kasdin

\author{
Department of Mechanical and Aerospace Engineering, Princeton University \\ ${ }^{\perp}$ Department of Astrophysical Sciences, Princeton University
}

\begin{abstract}
A new method is presented for amplitude and phase control using two liquid crystal spatial light modulators in conjunction with a white light Michelson interferometer. Preliminary proof-of-concept measurements are given showing the prospect of using this method for correction of amplitude errors in telescopes.
\end{abstract}

Keywords: Michelson interferometer, spatial light modulator, white light, amplitude correction, phase correction

\section{INTRODUCTION}

We are studying shaped-pupil coronagraphs for use in the direct imaging of earth-like planets around nearby stars. The shaped-pupil idea, first proposed by David Spergel (ref. 1), reduces the effects of diffraction from the parent star to improve potential visibility of faint planets.

Two telescope entrance pupils are shown in Figure 1 -- A circular pupil and a Spergel pupil.
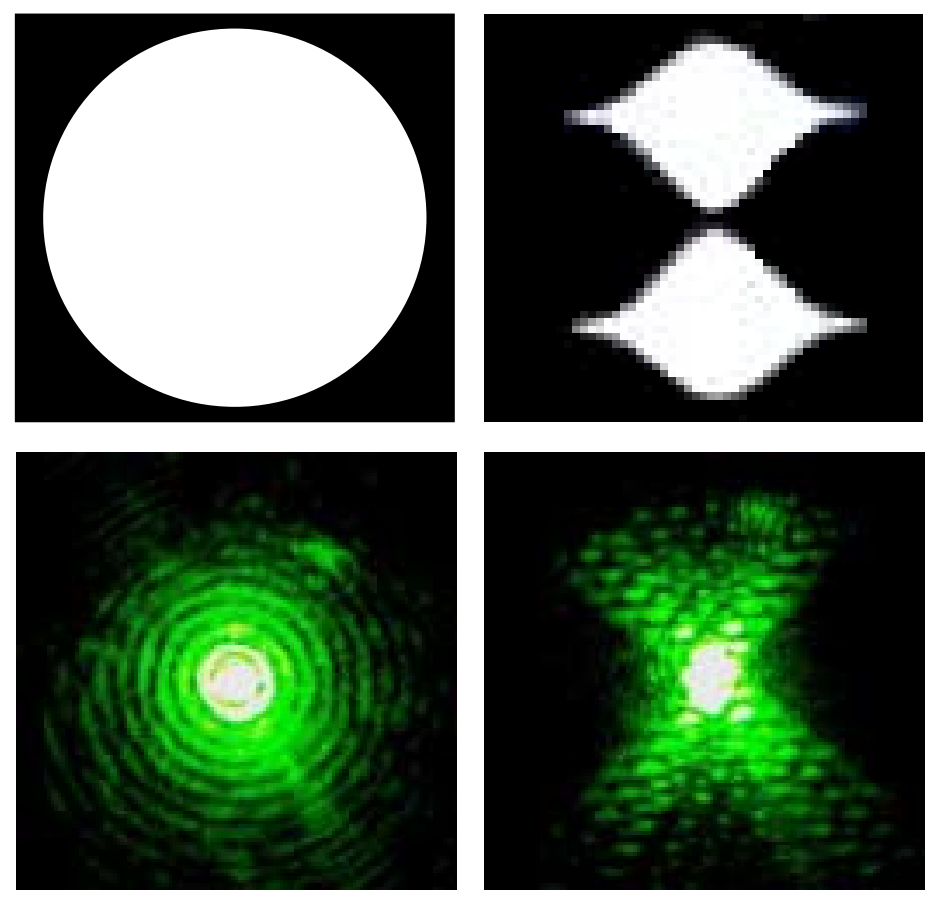

Figure 1. Telescope Pupils and Corresponding Focal Images

Also shown are focal images corresponding to the two pupils obtained using a laboratory prototype of a telescope in which a single-frequency HeNe laser $(543.4 \mathrm{~nm})$ serves as a plane wave source. A small parabolic reflector models the 
telescope primary. The Spergel pupil modifies the incoming field with a binary mask causing the optical field intensity along the horizontal-direction to assume a form that is roughly Gaussian. This Gaussian nature optimally reduces the light-spreading effects due to diffraction along the horizontal direction in the mirror's focal plane. This effect derives from the fact that a focusing mirror takes the spatial Fourier transform of the incoming pupil field when viewed in the focal plane. A familiar result that applies to a one-dimensional focusing mirror is that a "top hat" function maps to a "sin x / x" function while a Gaussian maps to a Gaussian. Note that the Gaussian ideal is not implemented precisely. A true Gaussian is infinite in extent. The "Gaussian" here is clipped or windowed. The windowed Gaussian is not optimal. The paper by Kasdin et al of this conference is a detailed discussion of pupil optimization.

Light from stars is broadband, not narrowband. The effect of the continuum of optical frequencies in starlight is to blur the oscillatory features resulting in the familiar point spread function that follows an overall amplitude envelope. One can imagine the point spread functions for the telescope with a circular aperture versus the shaped Spergel pupil aperture. The circular aperture displays a $1 / \rho$ envelope with circular symmetry about the peak of the focal spot. The Spergel aperture when blurred has a much tighter Gaussian envelope (along the horizontal-axis) and allows for two large dark triangular regions in the focal plane to the left and right of the peak of the focal spot. We refer to these triangular regions as our discovery space.

In order to obtain the $10^{10}$ contrast ratio that is needed to detect earth-like planets about the nearer stars, it is necessary that the optics of the telescope be exceptional. Indeed, the only practical way to obtain such quality is to correct the telescope for errors in both wavefront and reflectivity. Wavefront (phase) errors have been traditionally corrected using deformable mirrors, while reflectivity (amplitude) errors have most often been ignored. This paper proposes a new approach to correction of both amplitude and phase errors in the telescope by means of a zero path length Michelson interferometer and two deformable mirrors.

In our laboratory tests instead of deformable mirrors we are using nematic liquid crystal spatial light modulators. Several researchers have used spatial light modulators in conjunction with interferometers to correct optical distortion. (ref. 2) These modulators are more affordable than deformable mirrors. For purposes of proof of concept they are equivalent. Our liquid crystal spatial light modulator was from Boulder Nonlinear Systems, model 128N. Like deformable mirrors, spatial light modulators retard the phase by a fixed time delay that is independent of wavelength. The spatial light modulator achieves this through a controllable variation in index of refraction. Like deformable mirrors, the spatial light modulator is organized as an array of pixels. The modulator that we use is $128 \times 128$ elements with 128 levels of phase shift per pixel. The maximum phase retardation is greater than $700 \mathrm{~nm}$ or more than $2 \pi$ at the reddest wavelength that we consider. At $400 \mathrm{~nm}$ the corresponding maximum phase shift is greater than $3.5 \pi$ ( i.e., (7/4) $\mathrm{x} 2 \pi$ ). In contrast with deformable mirrors, spatial light modulators must use polarized light. The spatial light modulator has a fast and slow axis, with the index of refraction of only one axis being able to be controlled. All of our measurements have been carried out with the light linearly polarized along the controllable (vertical) axis.

To adjust amplitude, a Michelson interferometer is used as shown in Figure 2 below. The Michelson functions as a light valve that controls whether light will pass through the telescope to the camera or will be reflected back to the source. To demonstrate this we used a HeNe laser $(612 \mathrm{~nm})$ as the source of plane waves. The beam splitter in the interferometer is $50 \%-50 \%$. The neutral density filter (OD 0.3 , metal film) is required in the leg associated with the Piezo-controlled mirror to balance the intensity of the interfering beams. This is needed because the surface relectivity of the spatial light modulator is only $60 \%$. The compensator plate is needed for use with broadband light as discussed later in this paper. 


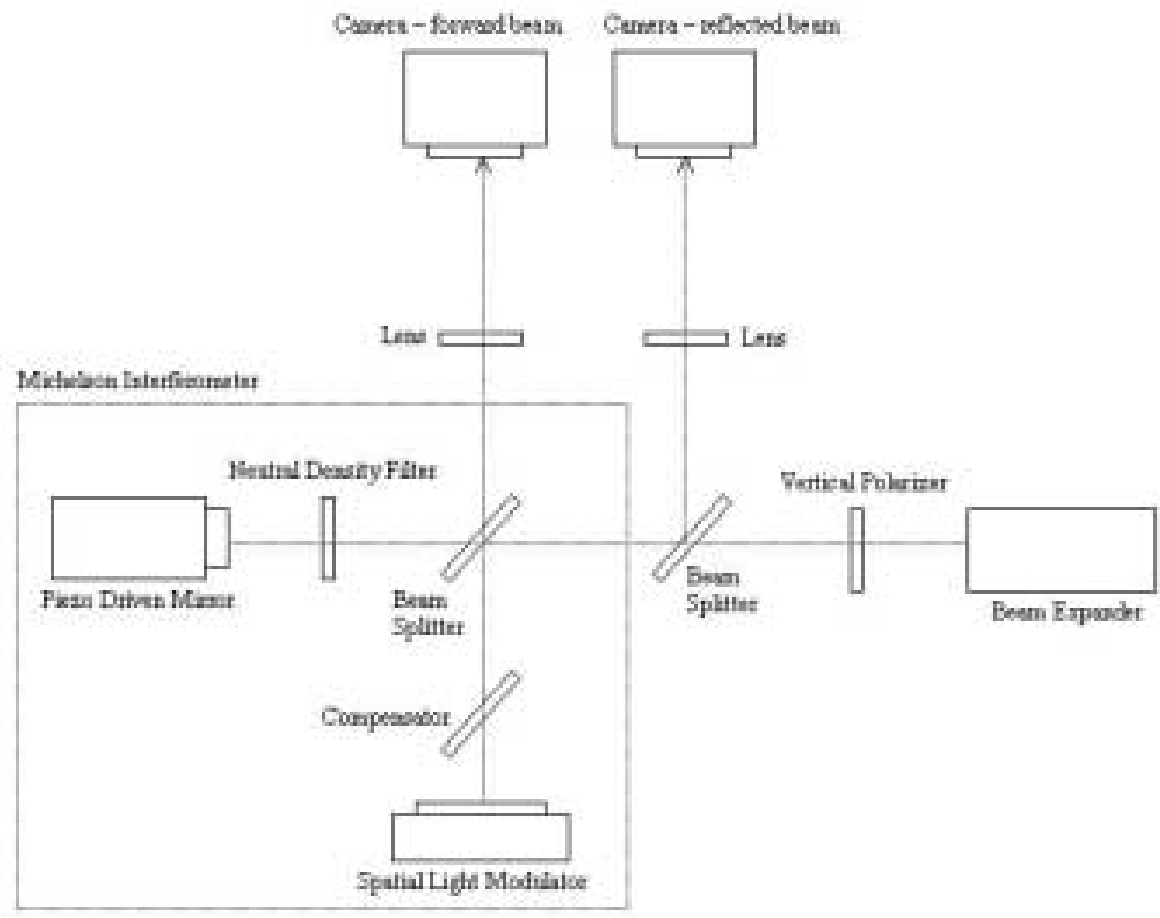

Figure 2. PLAN View of Test Bed

The control of amplitude is suggested in Figure 3. Here two sine waves of amplitude 0.5 (assume that incident plane wave source amplitude is 1) and differing phases are combined to achieve any desired amplitude. This is an idealization of the wave interference that occurs in both the forward and reflected beams of the interferometer. Shown in Figure 3 are phase differences of $0, \pi / 4, \pi / 2,3 \pi / 4$, and $\pi$. Note the reduction of amplitude as well as the shift in phase. The reduction in amplitude is desired - the shift in phase is not. A way to eliminate the phase shift is presented below. Recognize also that when the transmitted (forward) amplitude is reduced, the corresponding reflected wave (not shown in Figure 3) is increased such that the sum of transmitted and reflected beams equals the original incident field. This is required since the interferometer cannot create or destroy energy. We assume ideal (loss-less) reflectors. (In our laboratory study - broadband dielectric coatings are used for all reflective optics.) 


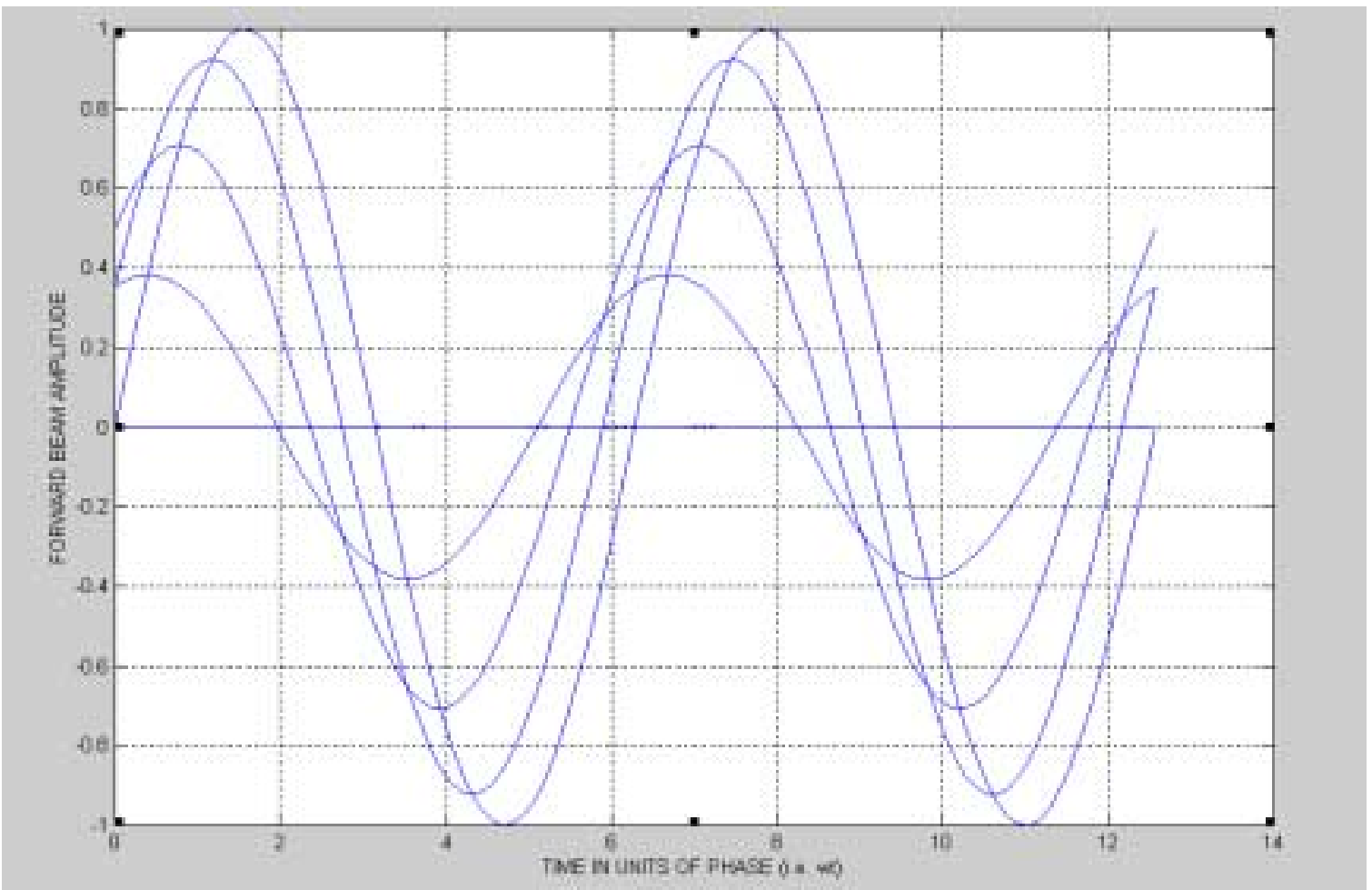

Figure 3. Forward Beam Amplitude for Spatial Light Modulator Pixel Phase of $0, \pi / 4, \pi / 2.3 \pi / 4$, and $\pi$.

Shown in Figure 4 are the Phase Shift and Amplitude obtained as a function of Spatial Light Modulator Pixel Phase.

The optical electric field amplitude is $\sqrt{\frac{1+\operatorname{COS}(\theta)}{2}}$ and the output phase is $\operatorname{TAN}^{-1}\left(\frac{\operatorname{SIN}(\theta)}{1+\operatorname{COS}(\theta)}\right)$. 


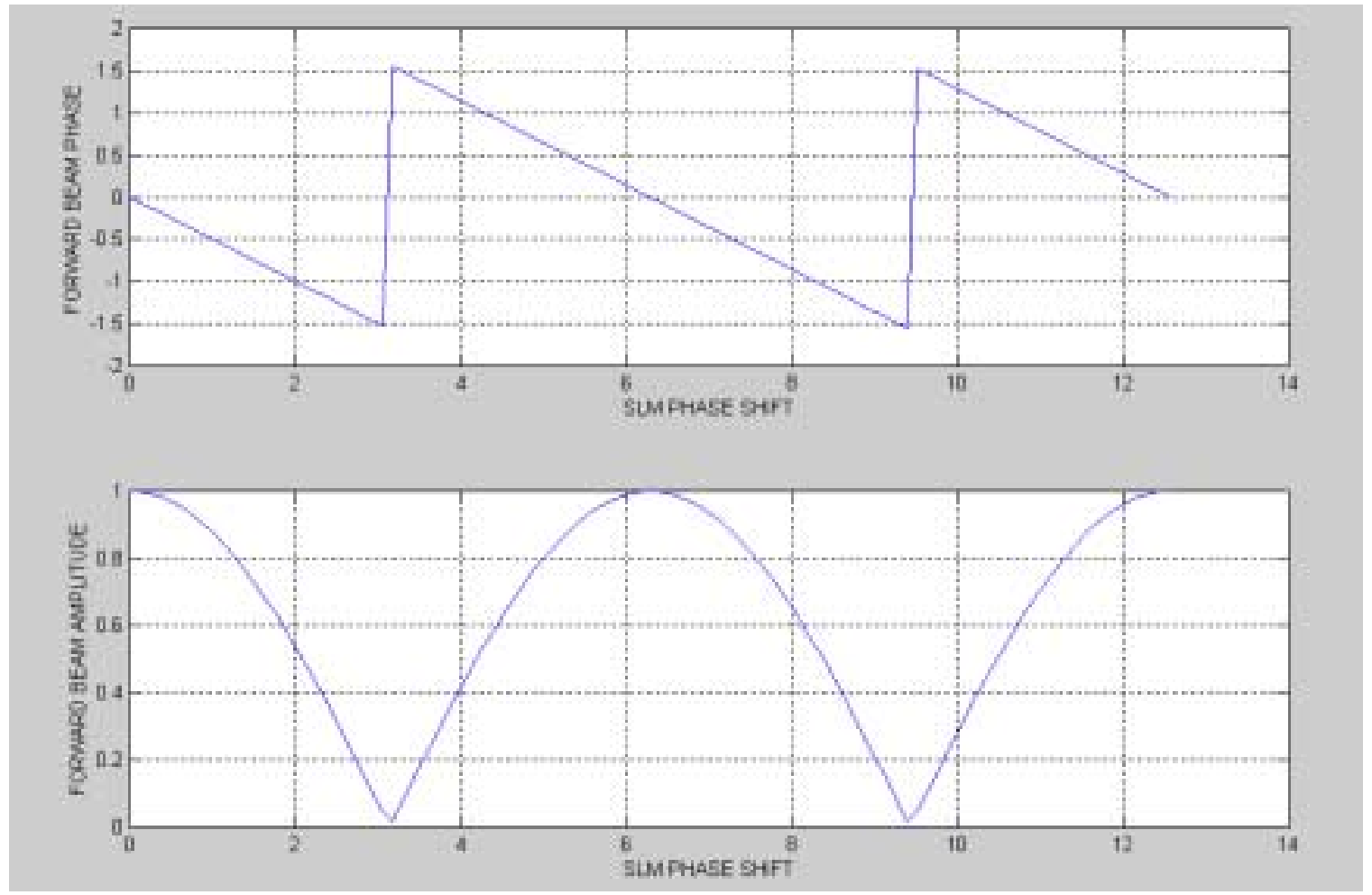

Figure 4. Phase and Amplitude of Forward Beam from Michelson versus Spatial Light Modulator Pixel Phase.

The control of amplitude here (with a single deformable mirror) unfortunately results in a phase shift. This phase shift can be eliminated by means of an additional spatial light modulator (to replace the Piezo Driven Mirror in Figure 2). In the dual spatial light modulator form, each pixel of one modulator is set to a desired positive phase shift (half of the total needed) and the corresponding pixel of the other modulator is set to a negative value of equal magnitude. The total phase shift of each pixel is therefore the difference of the two modulator values, whereas the forward beam phase shift is the average of the two legs which according to this positive / negative arrangement is zero.

This same arrangement can be used to correct for wavefront errors. Here one adjusts the phase of the both pixels in the same direction (positive or negative).

\section{SINGLE FREQUENCY TESTS - PROOF OF STEERING CONTROL ABILITY}

To prove that the interferometer can act as a valve causing light to either proceed forward or be back reflected based on the phase of each spatial light modulator pixel, we have performed a test using a $\mathrm{HeNe}(612 \mathrm{~nm})$ laser. Shown in Figure 5 are patterns from an intentionally misaligned Michelson. Each of these patterns records the face of the spatial light modulator $(5.1 \mathrm{~mm}$ square). The lenses in Figure 2 are used to image the face of the modulator onto each camera. In the central region a box is introduced that shifts the phase by $\pi$. The box uses about $20 \%$ of the pixels available. The image on the left is that obtained by the camera recording the forward beam. The image on the right is that obtained from the camera recording the reflected beam (refer to Figure 2). Note that the reflected beam is dimmer because half of the light is lost by the up-stream beam splitter. Notice most importantly that the patterns of light and dark are reversed in these two cameras. Areas that are dark in the forward camera are bright in the backward camera, and vice versa. This shows clearly how the modulator can be used to redirect light back to the source if needed on a pixel by pixel basis. Such a redirection may be used to make the forward beam uniform. We have not yet closed the loop to accomplish this feat. This is our next goal in this study. 

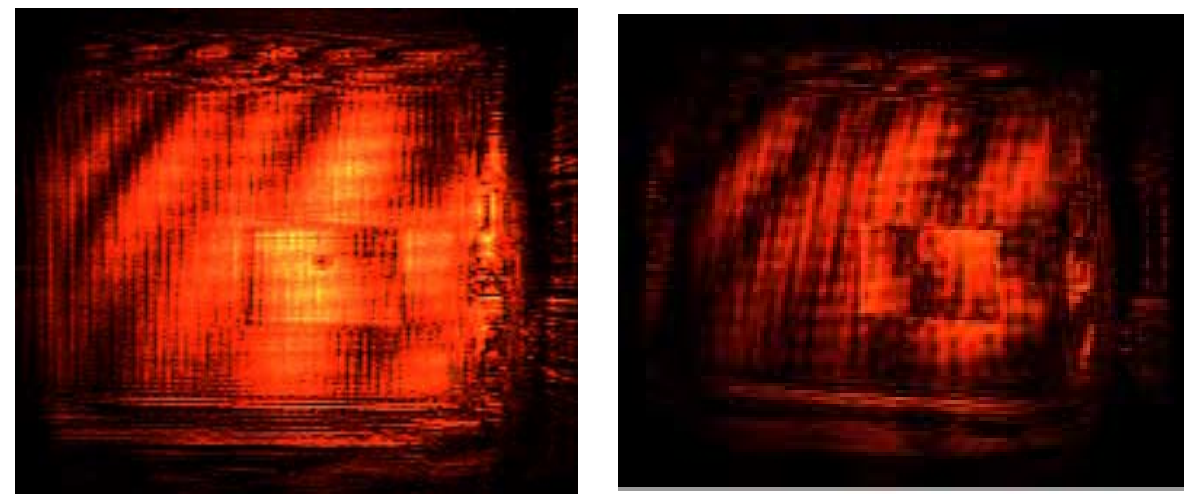

Figure 5. Camera Views of Forward and Backward Beams. Ideally the Two Patterns should sum to a Uniformly illuminated Square.

\section{WHITE LIGHT TESTS - PROOF OF BROADBAND CAPABILITY}

By using equal path length in both interferometer legs, it is possible to form fringes with white light. The fringe visibility is an indicator of interference pattern alignment. Such alignment is required if one intends for the light valve to function over a wide band of wavelengths. In addition, a compensator plate is added to the interferometer (see Figure 2) to balance the path length so that both legs of the interferometer pass through equal amounts of glass.

To show clearly that the equal path length concept can deal with white light we performed the following test. The laser in the previous section was replaced by an incandescent lamp. A small spot on the hot filament of the lamp was used to simulate a point of blackbody light. The Michelson was then very carefully adjusted so that the path length difference was within a few wavelengths of being equal. As mentioned previously, the indicator of equal length was fringe visibility. When the path length was not equal the camera recorded a featureless but illuminated patch. As the path length reached it equal point the fringes became more and more visible. In Figure 6 are patterns obtained in the forward beam with white light, where the position of the Spatial Light Modulator is such that maximizes fringe visibility. The Spatial Light Modulator is on a precision stage (New Focus, model XYZ) that allows for its translation. The five images shown correspond to five increasing values of phase shift of the box region relative to the surroundings. This is the same box region that was used in the previous test. Notice that it is possible to shift the pattern within the box, thereby clearly demonstrating that one can modify the amplitude of the transmitted field on a pixel by pixel basis. We did not record the backward beam but it is assumed to be the complement pattern to those obtained below. Note that the patterns below appear reddish. (Our two CCD cameras are color sensitive - Electrim Corp. Model 1000D). We think that the blue end of the spectrum was lost as the result of the dielectric beam splitter being used at an angle. We are in the process of obtaining new broadband metallic beam splitters and will determine experimentally the bandwidth of frequencies available for amplitude control. 

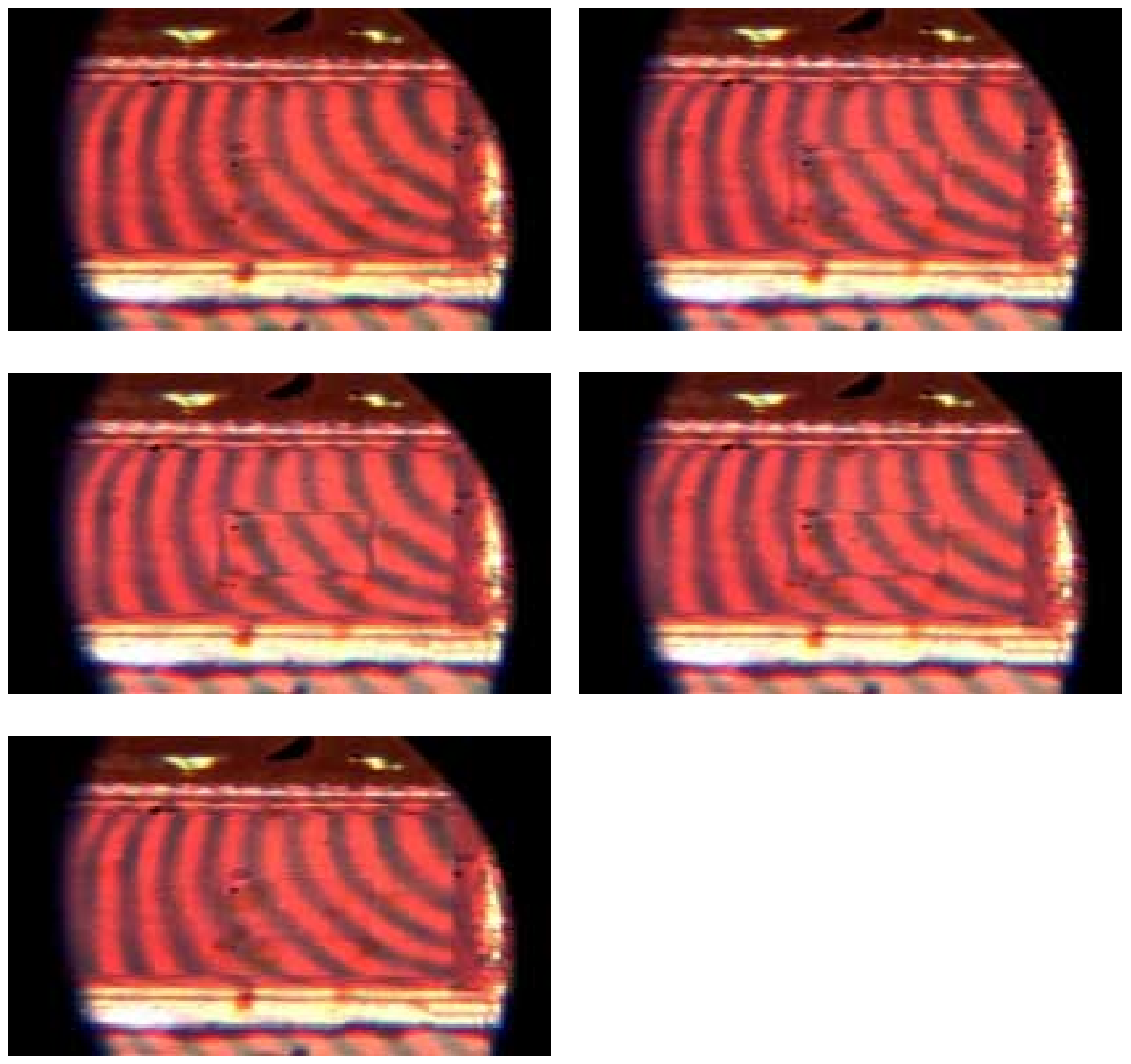

Figure 6. White Light Fringes and Their Shift in a Box Region using Spatial Light Modulator. This demonstrates white light amplitude control ability.

\section{CONCLUSION}

We have shown how a Michelson Interferometer incorporating a liquid crystal spatial light modulator may be used as a light valve to adjust the amplitude of a propagating optical field on a pixel by pixel basis. We have also demonstrated the ability of the instrument to work with white light when one adds the additional constraint of equal length interferometer legs.

Once we have obtained new monochrome cameras from Electrim as well as a shipment of new optics, we will proceed to close the loop and automatically adjust the amplitude of the forward beam to be uniform. We will then determine experimentally the amplitude control performance specifications. 
We would like to thank Scott Harris of the Air Force Research Laboratory and Teresa Ewing of Boulder Nonlinear Systems for their advice in controlling the Spatial Light Modulator using LabVIEW. We thank the NSF for seed support, and JPL and Princeton University for current support.

\section{REFERENCES}

1. For an early report of the Spergel Pupil see: M.G. Littman, J. Kasdin, and D. Spergel, "A Gaussian Pupil Coronagraph: A New Approach to Detecting Terrestrial Planets", American Astronomical Society Annual Meeting (Pasadena, CA), June 3 - 7 (2001).

2. See for example, R. Dou and M.K. Giles, "Closed Loop Adaptive Optics System using a LCTV as a Phase Retarder" Opt. Lett 20, 1583 (1995). 